\title{
Collisional Excitation Rates in the ISM
}

\author{
M. L. Dubernet ${ }^{1}$, P. Valiron ${ }^{3}$, F. Daniel ${ }^{1}$, A. Grosjean ${ }^{2}$, \\ F. Lique ${ }^{1}$, N. Feautrier ${ }^{1}$, A. Spielfiedel ${ }^{1}$, A. Faure ${ }^{3}$, \\ M. Wernli ${ }^{3}$, L. Wiesenfeld ${ }^{3}$, and C. Rist ${ }^{3}$ \\ ${ }^{1}$ LERMA, UMR CNRS 8112, Paris Observatory, 5 Place Janssen, 92195 Meudon, France \\ email: marie-lise.dubernet@obspm.fr \\ ${ }^{2}$ LAOB, Observatoire de Besançon, UMR CNRS 6091, Université de Franche-Comté, \\ 41 bis avenue de l'Observatoire, BP 1615, 25010 Besançon Cedex, France \\ ${ }^{3}$ Laboratoire d'Astrophysique de Grenoble, UMR 5571 CNRS-UJF, OSUG, \\ Université Joseph Fourier, BP 53, F-38041 Grenoble Cedex 9, France
}

\begin{abstract}
The paper focuses on collisional excitation rates of molecules by $\mathrm{He}$ and $\mathrm{H}_{2}$ relevant to the interstellar medium. It discusses currently available data, presents very recent work and outlines new work being carried out by various teams.
\end{abstract}

Keywords. ISM: molecules — molecular data — molecular processes

\section{Introduction}

Over the next few years, ground-based and space-based missions will open up the universe to high spatial and spectral resolution studies at infrared and submillimeter wavelengths. This will allow us to study, in much greater detail, the composition and the origin and evolution of molecules in space. These new missions can be expected to lead to the detection of many thousands of new spectral features. Identification, analysis and interpretation of these features in terms of the physical and chemical characteristics of the astronomical sources will require detailed astronomical tools supported by laboratory measurements and theoretical studies of chemical reactions and of collisional excitation rates on species of astrophysical relevance.

The present paper focuses on the latest theoretical studies on collisional excitation rate coefficients for molecules colliding with $\mathrm{H}_{2}$ and $\mathrm{He}$; these processes are important as they contribute to molecular excitation in competition with radiative processes. In cold environnements such as the interstellar medium, collisions with $\mathrm{H}_{2}$ are most important because of its high abundance, and collisions with He are often considered as a model for collisions with $\mathrm{H}_{2}$.

All the published rate coefficients used in astrophysical applications can be found on personal websites, such as those of S. Green (http://www.giss.nasa.gov/data/mcrates/) and D. Flower (http://ccp7.dur.ac.uk/) and in recent compilations, such as the Leiden Atomic and Molecular Database LAMDA (http://www.strw.leidenuniv.nl/ moldata/) by Schröier et al. (2005) and the BASECOL database (http://www.obspm.fr/basecol) by Dubernet et al. (2005). Those recent compilations have different approaches. The LAMDA database contains ready-to-use rate coefficients of the rotational excitation of molecules by $\mathrm{H}_{2}$ with extrapolations of rate coefficients in temperature and in transition, and connections of those rate coefficients to spectroscopic data from the JPL and CDMS catalogues. The BASECOL database contains all published rate coefficients and gives a complete information on the origin of the data with all relevant references. All 
the information given in the present paper on previous calculations is collected from the BASECOL database. The BASECOL database is a continuously evolving service that will offer standardized tools for manipulating fundamental rate coefficients and spectroscopic data and that will be included in the future theoretical services of the Virtual Observatory.

\section{Methodology}

Within the Born Oppenheimer approximation, the determination of these collisional rate coefficients requires two steps: the calculation of the interaction between nuclei and electrons for fixed nuclei, leading to so-called potential energy surfaces (PESs) and the scattering calculations. In the context of observations in the $\mathrm{mm}$ and submm range, the collisions concern regions with relatively low temperatures $(5 \mathrm{~K}<\mathrm{T}<1000 \mathrm{~K})$, there is no electronic excitation, nor any reactive channels, and the collisions generally involve a limited number of PESs describing a van der Waals system : a single PES for $\Sigma$ states and two surfaces for $\Pi$ states.

\subsection{Potential Energy Surfaces}

Recent progress in ab-initio quantum chemistry permits very accurate determinations of the PESs of small non-reactive systems. As the complexity of the calculations increases very steeply with the target accuracy, significant savings can be obtained using hierarchies of basis sets, correlation methods and meshes. By combining advanced treatments of both the electronic correlation and nuclear motion, a typical $\mathrm{cm}^{-1}$ accuracy can be achieved for intra-molecular PESs, as demonstrated by Polyansky et al. (2003) and Rajamaki et al. (2004) for vibrational investigations of $\mathrm{H}_{2} \mathrm{O}$ and $\mathrm{NH}_{3}$.

For inter-molecular PESs involving non-reactive molecules the coupled cluster theory with singles, doubles and perturbative triples, $\operatorname{CCSD}(\mathrm{T})$, is generally assumed to be very accurate. However, the effects resulting from theoretical levels beyond CCSD(T) are poorly known for such systems. From a comparison to experimental virial coefficients Jankowski \& Szalewicz (2005) estimated they might amount to several $\mathrm{cm}^{-1}$ in the vicinity of the $\mathrm{CO}-\mathrm{H}_{2}$ minimum. Basis set superposition errors are sizeable even for the largest basis set sizes, and should be removed by counterpoise. Various techniques can be used to approximate the infinite basis set limit at the $\operatorname{CCSD}(\mathrm{T})$ level of theory. A popular approach is to associate bond functions to large augmented correlation basis sets in order to improve the description of the wavefunction between the interacting molecules. This approach may be complemented with basis set extrapolation, as in Jankowski \& Szalewicz (2005) and references therein.

An alternative method is to describe properly the electron-electron correlation cusp that is responsible for the slow convergence of all conventional approaches with respect to the basis set size. Such explicitly correlated methods offer a direct way of reaching the basis set limit values within a single calculation, i.e. without extrapolation. In particular the so-called $\operatorname{CCSD}(\mathrm{T})-\mathrm{R} 12$ approach is computationally practical and proven to be highly accurate, in particular using adequate R12-suited basis sets. Appropriate references can be found in Faure et al. (2005a). Such CCSD(T)-R12 calculations can also be used to monitor the accuracy of conventional PESs, as was done for $\mathrm{He}$ and $\mathrm{H}_{2}$ interacting with $\mathrm{CO}, \mathrm{NH}_{3}, \mathrm{HF}$ and $\mathrm{HC}_{3} \mathrm{~N}$ in Wernli et al. (2005), Valiron (unpublished) and Wernli et al. (2006). 


\subsection{Scattering Calculations}

Quantum close-coupling calculations are feasible in the low energy range and lead to nearly exact results. Calculations can be extended to higher energies using approximate quantum or semi classical approaches. The fast and popular Infinite Order Sudden (IOS) quantum approximation usually converges well whenever the relevant $\Delta j$ energies are small in comparison to the available collisional energy. When the energy is augmented, this condition is fulfilled for the lower- $j$ levels, but not for the highest- $j$ levels. For vibrational quenching or excitation rates, the corresponding VCC-IOS approximation may also prove questionable for molecule-molecule pairs involving large rotational constants, as pointed out by Krems et al. (2001) and Faure et al. (2005b). In that respect the extension of present scattering calculations to the obtaining of inelastic rates for warm gas or shocked regions may be problematic, if accurate results are sought.

\section{New Results}

\section{1. $\mathrm{H}_{2} \mathrm{O}+\mathrm{H}_{2}$}

The available excitation rate coefficients of $\mathrm{H}_{2} \mathrm{O}$ by $\mathrm{H}_{2}$ are those calculated by Phillips et al. (1995) and Phillips et al. (1996), using the close-coupling and the coupled states methods with the potential energy surface (PES) calculated by Phillips et al. (1994). Those authors provided data between $20 \mathrm{~K}$ and $140 \mathrm{~K}$ for a number of ortho/para $\mathrm{H}_{2} \mathrm{O}$ - ortho/para $\mathrm{H}_{2}$ pure rotational transitions. These calculations were extended to $5 \mathrm{~K}$ by Dubernet et al. (2002) and Grosjean et al. (2003), using pure close-coupling calculations over the same PES (Phillips et al. 1994). The results presented in Dubernet et al. (2002) were significantly different from those presented earlier by Phillips et al. (1996) at $20 \mathrm{~K}$. In particular, the excitation rate coefficient for the $1_{01}-1_{10}$ transition of water was more than $50 \%$ larger than in Phillips et al. (1996). This was a consequence of an insufficient fine energy grid used by Phillips et al. (1996) in integrating the cross-sections over a Maxwellian distribution of kinetic energies.

A new 9D PES of the $\mathrm{H}_{2}-\mathrm{H}_{2} \mathrm{O}$ system has recently been calculated by Faure et al. (2005a) using a three-step procedure: (i) a 5D rigid-rotor PES reference was computed at the $\operatorname{CCSD}(\mathrm{T})$ level of theory; (ii) this reference surface was then calibrated to few $\mathrm{cm}^{-1}$ accuracy using 812 "high cost" CCSD(T)-R12 calculations; (iii) the R12-corrected rigid rotor surface was then extended to $9 \mathrm{D}$ using a new set of $\operatorname{CCSD}(\mathrm{T})$ calculations. The resulting 9D PES contains all relevant information to describe the interaction of all $\mathrm{H}_{2} \mathrm{O}-\mathrm{H}_{2}$ isotopomers in zero-point or excited bending states. As a first application, high temperature $(1500<\mathrm{T}<4000 \mathrm{~K})$ rate constants for the relaxation of the $\nu_{2}$ bending mode of $\mathrm{H}_{2} \mathrm{O}$ were estimated from quasiclassical trajectory calculations by Faure et al. (2005a) and the role of rotation in the vibrational relaxation of water is emphasized by Faure et al. (2005b). Their results are larger by an order of magnitude than those currently used in the astrophysical literature and might lead to a thorough reinterpretation of vibrationally excited water emission spectra from space.

A 5D PES was also obtained by averaging the 9D PES over the ground vibrational states of $\mathrm{H}_{2} \mathrm{O}$ and $\mathrm{H}_{2}$. Using this averaged 5D PES, new scattering calculations were carried out for the rotational excitation of $\mathrm{H}_{2} \mathrm{O}(000)$ by $\mathrm{H}_{2}(\mathrm{v}=0)$ using the same procedure as in Dubernet et al. (2002) and Grosjean et al. (2003) with a larger basis set as reported in Daniel et al. (2006). The rates involving ortho- $\mathrm{H}_{2}$ are changed by 10 to $20 \%$ only, while low energy para- $\mathrm{H}_{2}$ rates may differ by a factor of 2 or more.

These changes reflect the improvements in the PES, where surprisingly the largest contribution is attributed to the vibrational averaging. However Valiron et al. (2006a) 
showed that the vibrationally averaged PES can be accurately approximated by rigid rotor calculations using properly vibrationally averaged geometries for both interacting molecules, as already postulated by Mas \& Szalewicz (1996) and Jeziorska et al. (2000). This simplification may facilitate accurate PES calculations whenever inelastic vibrational effects are not to be considered. An application is underway for $\mathrm{H}_{2} \mathrm{CO}-\mathrm{H}_{2}$ by Valiron (2006b).

\section{2. $\mathrm{N}_{2} \mathrm{H}^{+}+\mathrm{He}$}

Up until now, the only available rate coefficients for $\mathrm{N}_{2} \mathrm{H}^{+}$excited by $\mathrm{He}$ were pure rotational excitation rate coefficients among rotational levels $j<6$ and for temperature up to $40 \mathrm{~K}$. There were calculated by Green (1975), using an electron gas model for the potential energy surface. Using an adiabatic decoupling approximation a two-dimensional potential energy surface corrected for the influence of the $\nu_{1}$ (mainly NH-local mode) stretching vibration was calculated by Meuwly \& Bemish (1997) at the CCSD(T)/augcc-pVQZ level. Its reliability has been assessed by comparing energies of bound states and rotational constants with experimental data (Meuwly et al. 1996). The percentage differences between earlier rotational results by Green (1975) and new calculations by Daniel et al. (2005) are larger for transitions with large $\Delta j$ and vary in the range from a few percent to $100 \%$. In overall the new rates are larger for all transitions and the differences decrease with increasing temperature.

In astrophysical applications only the splittings due to couplings with the nuclear spins of the two nitrogen atoms are resolved and there are 9 hyperfine components for a given rotational level $j>0$. The cross sections between hyperfine levels are obtained using a recoupling technique, first introduced by Corey \& McCourt (1983) for the case of a single electronic spin and extended in Daniel et al. (2004) to the case of 2 nuclear spins. The dynamical problem reduces to spin-less equations, which is solved using close-coupling (CC) methods. It is found that the only well-defined propensity rule among hyperfine rate coefficients is $\Delta F=\Delta F_{1}=\Delta j$ and that calculations are required in order to obtain the relative intensities of the 2 spin hyperfine rate coefficients at temperature below $50 \mathrm{~K}$. Daniel et al. (2005) note that the usual simple approaches such as Infinite Order Sudden scaling and proportionality of the hyperfine rate coefficients to the final degeneracy of the hyperfine levels are inadequate. The authors provide Boltzmann averages of the so-called opacity factors; these allow calculation of pure rotational rate coefficients, rate coefficients among hyperfine levels as well as among magnetic sublevels of hyperfine levels for the 7 first rotational levels and for temperature less than $50 \mathrm{~K}$.

These new results have been used in astrophysical applications (Daniel et al. 2005; Daniel et al. 2006a) and scattering calculations are currently extended to reach a temperature of $300 \mathrm{~K}$. The $\mathrm{N}_{2} \mathrm{H}^{+}+\mathrm{H}_{2}$ system is currently under investigation by M. Meuwly and M.L. Dubernet.

\section{3. $\mathrm{SO}+\mathrm{He}$}

The previous available rate coefficients were provided for the $\mathrm{SO}-\mathrm{H}_{2}$ system by Green (1994) at temperatures ranging from $50 \mathrm{~K}$ to $350 \mathrm{~K}$ among the 70 lowest fine-structure energy levels corresponding to a maximal rotational level $N_{\max }=23$. The author used a CS- $\mathrm{H}_{2}$ PES, which had been adapted (Green 1975) from an electron gas model (Gordon et al. 1972) of CS-He, treating the CS molecule as a rigid rotor. The long range part of the CS-He PES had been modified to account for the electrostatic interaction between $\mathrm{CS}$ and $\mathrm{H}_{2}$. The rate coefficients among fine-structure levels were obtained with an IOS scaling relationship similar to the one used by Neufeld \& Green (1994) for $\mathrm{HCl}+\mathrm{He}$. 
New collisional excitation cross sections of the fine-structure levels of SO colliding with He have recently been calculated by Lique et al. (2005a) at low energies using a full close-coupling treatment and a new ab initio potential energy surface calculated at the $\operatorname{CCSD}(\mathrm{T})$ level with an aug-cc-pVQZ basis set complemented by bond functions. Rate coefficients were obtained for temperatures ranging from $5 \mathrm{~K}$ to $50 \mathrm{~K}$. The results displayed the expected propensity rules $\Delta J=\Delta N$. The use of recoupling techniques from spin-free cross sections was investigated at low energy. This approximation is not valid for excitation between the first levels where the fine structure splitting is large compared to the rotational splitting, but it should provide a reasonable estimate of rates for high- $J$ levels at large temperatures. Some preliminary tests by Lique et al. (2005b) show that the use of the new rate coefficients compared to the use of rate coefficients previously calculated by Green (1994) can significantly change the diagnostics of the SO abundance in dark clouds. Calculations for temperatures up to $300 \mathrm{~K}$ are under way.

\section{4. $\mathrm{HC}_{3} \mathrm{~N}+\mathrm{He} / \mathrm{H}_{2}$}

The currently available rate coefficients are those of Green \& Chapman (1978); they are calculated among the 21 lowest rotational levels for $\mathrm{T}=10,20,40,80 \mathrm{~K}$ using quasiclassical calculations combined with the Infinite Order scaling relationship. Their PES is computed with an electron gas model PES (Gordon et al. 1972), treating the $\mathrm{HC}_{3} \mathrm{~N}$ molecule as a linear rigid rotor, with bonds fixed at experimental values.

Two new PESs have been calculated at a $\operatorname{CCSD}(\mathrm{T})$ level for the $\mathrm{HC}_{3} \mathrm{~N}-\mathrm{He}$ and $\mathrm{HC}_{3} \mathrm{~N}-$ $\mathrm{H}_{2}$ systems by Wernli et al. (2006). The new $\mathrm{HC}_{3} \mathrm{~N}-\mathrm{He}$ agrees pretty well with the PES obtained by Akin-Ojo et al. (2003). Steric hindrance problems involving the $\mathrm{HC}_{3} \mathrm{~N}$ rod limit the convergence of the angular expansion of the PES, as anticipated by Green \& Chapman (1978). However for low energy collisions Wernli et al. (2006) showed it is feasible to regularize the PES by smoothing out the repulsive walls and to achieve a perfectly converged angular expansion. Corresponding close-coupling calculations led to surprising results due to the rod-like features of the PES. Firstly quantum interferences strongly defavour odd $\Delta j$ transitions and favour even $\Delta j$ ones. This propensity rule is likely to favour the $J=1$ population for $\mathrm{H}_{2}$ densities in the $10^{3}-10^{4} \mathrm{~cm}^{-3}$ range. Secondly, despite the very large $\mathrm{HC}_{3} \mathrm{~N}$ dipole moment, the para- $\mathrm{H}_{2}$ and ortho- $\mathrm{H}_{2}$ rates are nearly identical. While the even $\Delta j$ propensity rule could not be found in the quasiclassical calculations by Green \& Chapman (1978), the new rates remain within the same order of magnitude despite the very crude electron gas model PES. This is not too surprising as the rod-like features of the PES dominate the scattering.

\section{5. $\mathrm{CO}+\mathrm{H}_{2}$}

Rotational rate coefficients among the lowest 29 levels of $\mathrm{CO}$ with para- $\mathrm{H}_{2}(j=0)$ and the lowest 20 levels of $\mathrm{CO}$ with ortho- $\mathrm{H}_{2}(j=1)$ were provided using the close-coupling method by Flower (2001) for temperatures ranging from $5 \mathrm{~K}$ to $400 \mathrm{~K}$. Those calculations were of better quality and superseded the results of Mengel et al. (2001). It should be noted that both Flower and Mengel used the PES of Jankowski \& Szalewicz (1998), but Mengel used a scaled version of the PES, while Flower used it without modifications. Quantum mechanical studies of this system had previously been undertaken by Green \& Thaddeus (1976), who used a scaled CO-He interaction PES, and by Flower \& Launay (1985) and Schinke et al. (1985), who used a different ab initio CO- $\mathrm{H}_{2}$ PES.

The Jankowski \& Szalewicz (1998) PES has recently been improved by Jankowski \& Szalewicz (2005) and new close-coupling calculations were carried out for the rotational excitation of $\mathrm{CO}$ by $\mathrm{H}_{2}$ (see Wernli et al. 2005). The authors confirmed the quality of the PES by independent CCSD(T)-R12 calculations, and found that a $10 \%$ difference 
in the PES brings about a $30 \%$ to $50 \%$ difference in the final rate coefficients at low temperature compared to the earlier results by Flower (2001). They also reported fair agreement above a temperature of $70 \mathrm{~K}$.

\section{6. $\mathrm{NH}_{3}+\mathrm{He}$}

Numerous studies have been carried out on the rotational excitation of $\mathrm{NH}_{3}$. The available set of data concerns the rotational excitation of $\mathrm{NH}_{3}$ by He, obtained by Green (1981) using coupled states calculations and a PES of Davis et al. (1979).

A new set has been calculated by Machin \& Roueff (2005) using rigid-body closecoupling calculations (no inversion) with a PES by Hodges \& Wheatley (2001). They report de-excitation rate coefficients for transitions up to $j=4$ for para- $\mathrm{NH}_{3}$, and up to $j=7$ for ortho- $\mathrm{NH}_{3}$ for temperatures between $5 \mathrm{~K}$ and $300 \mathrm{~K}$. Large differences are obtained with the previous results of Green (1981), mainly attributed to the PES changes. Comparisons with experiments fare well when the rate coefficients are summed over the 2 final parity components. It is believed that the discrepancies with experimental results come from the neglect of the inversion motion.

Interaction energies were checked against $\operatorname{CCSD}(\mathrm{T})$-R12 calculations by Valiron (unpublished) for several interacting geometries and intermolecular separations. The PES by Hodges \& Wheatley (2001) is slightly too repulsive. Agreement is better along the attractive orientations, with a $2 \mathrm{~cm}^{-1}$ offset for the absolute minimum, and poorer for the less attractive orientations with a typical $5 \mathrm{~cm}^{-1}$ offset at 7 bohrs, to be compared to the $\sim 35 \mathrm{~cm}^{-1}$ absolute minimum. As the scattering is expected to be most sensitive to the minima and to the positioning of the repulsive walls this surface should provide a fair accuracy for astrophysical applications. PES and rate calculations involving the dominant collision partner $\mathrm{H}_{2}$ are planned within the Molecular Universe European network.

\section{7. $\mathrm{HF}+\mathrm{He}$}

Various studies have been carried out on ro-vibrational excitation of HF by He. The latest study involves the calculation of a new PES by Stoecklin et al. (2003) at a CCSD(T) level with an aug-cc-pVQZ basis set complemented by bond functions. Interaction energies for both the primary and secondary minima were checked against CCSD(T)-R12 calculations by Valiron (unpublished) and agreed to within $0.3 \mathrm{~cm}^{-1}$. This extremely accurate PES by Stoecklin et al. (2003) was subsequently used for close-coupling calculations of the rotational excitation of HF by Reese et al. (2005). The authors provide rotational deexcitation rate coefficients for levels up to $j=9$ and for temperatures up to $300 \mathrm{~K}$. It should be noted that Moszynski et al. (1994) and Moszynski et al. (1996) had provided a SAPT PES that agreed well with a semi-empirical PES of Lovejoy \& Nesbitt (1990) obtained from near-infrared spectroscopy.

The predicted rates are small and lead to a high critical density, in agreement with the modeling of the pioneering $\mathrm{HF}_{2-1}$ observations in absorption towards Sgr-B2 by Neufeld et al. (1997). However due to the large dipole moment of HF the collisional rates with ortho- $\mathrm{H}_{2}$ might be substantially higher.

\section{Conclusions}

Accurate predictions of collisional excitation rates are now feasible for a large set of small-molecule interactions with $\mathrm{He}$ and $\mathrm{H}_{2}$.

The accuracy of the potential energy surfaces (PES) can be monitored at a given level of theory using basis set extrapolation techniques or preferably by explicitly correlated approaches, and few $\mathrm{cm}^{-1}$ accuracy is now attainable for small non-reactive molecules. 
Ideally, the PES should be averaged over the relevant vibrational states of both interacting molecules. However it is sufficient to perform rigid rotor calculations using vibrationally averaged geometries. Recent investigations using new PESs for $\mathrm{CO}, \mathrm{H}_{2} \mathrm{O}$, $\mathrm{NH}_{3}, \mathrm{~N}_{2} \mathrm{H}^{+}$, SO and $\mathrm{HC}_{3} \mathrm{~N}$ permitted the estimation of the influence of PES errors on the final inelastic rates. Except at very low temperature or for the smallest rates the error amplification is moderate, generally much below an order of magnitude. Consequently accurate PESs with a few $\mathrm{cm}^{-1}$ accuracy should lead to $5-10 \%$ accuracy in the rates if appropriately converged and energy-averaged close-coupling calculations can be performed. However that level of PES accuracy is highly computer-time consuming. It requires an optimized choice of the grid geometries for the ab initio calculations and performance of an analytic fit of the PES leading to some additional inaccuracy.

Moreover the best level of accuracy is out of reach of current approximate approaches for scattering. Accordingly, the main source of uncertainties may come in the future from the scattering calculations instead of the PES, especially for higher energies or inelastic ro-vibrational rates for which brute force close-coupling calculations are not feasible, especially as mixing between electronic states may occur.

On the other hand, astronomers are welcome to express their needs in terms of accuracy, as much cheaper PESs would be sufficient to obtain moderate rate accuracies.

\section{Acknowledgements}

The authors thank E. Roueff, L. Machin, and T. Stoecklin for providing information on their results. New results presented here illustrate the French ongoing developments supported by the CNRS program "Physique et Chimie du Milieu Interstellaire" and by the "Molecular Universe" European network (2005-2008). Most calculations benefited from substantial time allocations on the French supercomputers IDRIS and CINES.

\section{References}

Akin-Ojo, O., Bukowski, R., \& Szalewicz, K. 2003, J. Chem. Phys. 119, 8379

BASECOL database, http://www.obspm.fr/basecol

Corey, G. C. \& McCourt, F. R. 1983, J. Phys. Chem. 87, 2723

Daniel, F., Dubernet, M.-L., \& Meuwly, M. 2004, J. Chem. Phys. 121, 4540

Daniel, F., Dubernet, M.-L., Meuwly, M., Cernicharo, J., \& Pagani, L. 2005, MNRAS, in press

Daniel, F., Cernicharo, J., \& Dubernet, M.-L. 2005, Semaine de l'Astrophysique Française, meeting held in Strasbourg, France, Eds.: F. Casoli, T. Contini, J. M. Hameury and L. Pagani. EdP-Sciences, Conference Series, in press

Daniel, F., Dubernet, M.-L., Grosjean, A., Faure, A., Valiron, P., Wernli, M., Wiesenfeld, L., Rist, C., Noga, J., \& Tennyson, J. 2006, A\&A to be submitted

Daniel, F., Cernicharo, J., \& Dubernet, M.-L. 2006, Ap. J. to be submitted

Davis, S. L., Boggs, J. E., \& Mehrotra, S. C. 1979, J. Chem. Phys. 71, 1418

Dubernet, M.-L. \& Grosjean, A. 2002, A\& A 390, 793

Dubernet, M.-L., Grosjean, A., Flower, D., Roueff, E., Daniel, F., Moreau, N., \& Debray, B. 2005 J. Plasma Fusion Research Series, volume 7

Faure, A., Valiron, P., Wernli, M., Wiesenfeld, L., Rist, C., Noga, J., \& Tennyson, J. 2005, J. Chem. Phys., 122, 221102

Faure, A., Wiesenfeld, L., Wernli, M., \& Valiron, P. 2005b, J. Chem. Phys. 123, 104309

Flower, D. R. 2001, J. of Phys. B 34, 2731

Flower, D. R. \& Launay, J.-M. 1985, MNRAS 214, 271

Gordon, R. G. \& Kim, Y. S. 1972, J. Chem. Phys. 56, 3122

Green, S. 1975, Ap. J. 201, 366

Green, S. 1981, NASA Technical Memorandum 83869 
Green, S. 1994, Ap. J. 434, 188

Green, S. \& Chapman, S. 1978, Ap. J. Suppl. 37, 169

Green, S. \& Thaddeus, P. 1976, Ap. J. 205, 766

Grosjean, A., Dubernet, M.-L., \& Ceccarelli C. 2003, A\&A, 408, 1197

Hodges, M. P. \& Wheatley, R. J. 2001, J. Chem. Phys. 114, 8836

Jankowski, P. \& Szalewicz, K. 1998, J. Chem. Phys. 108, 3554

Jankowski, P. \& Szalewicz, K. 2005, J. Chem. Phys. 123, 104301

Jeziorska, M., Jankowski, P., Szalewicz, K., \& Jeziorski, B. 2000, J. Chem. Phys. 1132957

Krems, R. V., Marković, N., Buchachenko, A. A., \& Nordholm, S. 2001, J. Chem. Phys. 114, 1249

Lique, F., Spielfiedel, A., Dubernet, M.-L., \& Feautrier, N. 2005, J. Chem. Phys., in press

Lique, F., Spielfiedel, A., Dubernet, M.-L., \& Feautrier, N. 2005, Semaine de l'Astrophysique Française, meeting held in Strasbourg, France, Eds.: F. Casoli, T. Contini, J.M. Hameury and L. Pagani. EdP-Sciences, Conference Series, in press

Lovejoy, C. M. \& Nesbitt, D. J. 1990, J. Chem. Phys. 93, 5387

Machin, L. \& Roueff, E. 2005, J. of Phys. B 38, 1519

Mas, E. M. \& Szalewicz, K. 1996, J. Chem. Phys. 104, 7606

Mengel, M., de Lucia, F. C., \& Herbst, E. 2001, Can. J. of Phys. 79, 589

Meuwly, M. \& Bemish, R. J. 1997, J. Chem. Phys. 106, 8672

Meuwly, M., Nizkorodov, S. A., Maier, J. P., \& Bieske, E. J. 1996, J. Chem. Phys. 104, 3876

Moszynski, R., de Weerd, F., Groenenboom, G. C., \& van der Avoird, A. 1996, Chem. Phys. Lett. 263, 107

Moszynski, R., Wormer, P. E. S., Jeziorski, B., \& van der Avoird, A. 1994, J. Chem. Phys. 101, 2811

Neufeld, D. A. \& Green, S. 1994, Ap. J. 432, 158

Neufeld, D. A., Zmuidzinas, J., Schilke, P., \& Phillips, T. G. 1997, A\&A 488, L141

Phillips, T. R., Maluendes, S., \& Green, S. 1995, J. Chem. Phys. 102, 6024

Phillips, T. R., Maluendes, S., \& Green, S. 1996, Ap. J. Suppl. 107, 467

Phillips, T. R., Maluendes, S., McLean, A. D., \& Green, S. 1994, J. Chem. Phys. 101, 5824

Polyansky, O. L., Csaszar, A. G., Shirin, S. V., Zobov, N. F., Barletta, P., Tennyson, J., Schwenke, D. W., \& Knowles, P. J. 2003, Science 299, 539

Rajamaki, T., Kallay, M., Noga, J., Valiron, P., \& Halonen, L. 2004, Mol. Phys. 102, 2297

Reese, C., Stoecklin, T., Voronin, A., \& Rayez, J. C. 2005, A\&ऽA 430, 1139

Schinke, R., Engel, V., Buck, U., Meyer, H., \& Diercksen, G. H. F. 1985, Ap. J. 299, 939

Schöier, F. L., van der Tak, F. F. S., van Dishoeck, E. F., \& Black, J. H. 2005, A\&BA 432, 369

Stoecklin, T., Voronin, A., \& Rayez, J. C. 2003, Chem. Phys. 294, 117

Valiron, P., Wernli, M., Faure, A., \& Rist, C. 2006, J. Chem. Phys., in preparation

Valiron, P. 2006b, J. Chem. Phys., in preparation

Valiron, P., unpublished

Wernli, M., Valiron, P., Faure, A., Wiesenfeld, L., Jankowski, P., \& Szalewicz, K. 2005, A\& A, in press

Wernli, M., Valiron, P., Faure, A., \& Wiesenfeld, L. 2006, A\&\&A, in preparation

\section{Discussion}

TOwnes: Are there any experimental results, which directly test your calculations of cross-sections, and if so how well do they agree?

DubERnET: No there are, unfortunately, no direct experimental tests as yet. 